\title{
Avulsão dental como resultado de trauma: o grau de conhecimento de profissionais socorristas
}

\author{
Tooth avulsion from trauma: the level of knowledge \\ of emergency physicians
}

\author{
Rodrigo José Beretta* \\ Maria Eduarda Santos Volpato** \\ Dayani Galato*** \\ Gláucia Helena Faraco de Medeiros ${ }^{* * *}$
}

\section{Resumo}

Objetivo: avaliar o grau de conhecimento dos profissionais socorristas a respeito do atendimento nos casos de avulsão e reimplante dental. Sujeitos e método: estudo transversal realizado por meio de entrevistas com socorristas profissionais que trabalham em serviço de atendimento de urgência de uma região de Santa Catarina. Resultados: foram entrevistados 101 colaboradores dos quais $74,3 \%$ (75) afirmaram que já atenderam casos de trauma dental e $52,5 \%$ (53) de avulsão dental, entretanto apenas $38,6 \%$ (39) referiram ter recebido alguma orientação acerca de trauma dental e 20,8\% (20) sobre avulsão durante sua formação. Dos entrevistados $74(73,3 \%)$ possuem conhecimento de que um dente avulsionado pode ser reimplantado, apenas $19(18,8 \%)$ dos entrevistados afirmaram que o elemento deve ser irrigado e colocado em solução salina ou na boca do paciente, $69(68,3 \%)$ indicaram alguma forma de armazenar o dente avulsionado. Conclusão: o conhecimento dos profissionais que trabalham no serviço de atendimento de urgência estudado quanto ao atendimento de pacientes que sofreram trauma dental, é insuficiente para o cuidado adequado dos mesmos.

Palavras-chave: Traumatismos Faciais. Traumatismos Dentários. Avulsão Dentária. Reimplante Dentário.

\section{Introdução}

O trauma pode ser definido como o conjunto das perturbações causadas subitamente por um agente físico de etiologia, natureza e extensão variadas, podendo interferir nos diferentes segmentos corpóreos. Estas perturbações podem ocorrer com uma frequência importante na pele da face, nos ossos faciais e nos dentes por estarem mais expostos às agressões devido à sua projeção corporal anterior ${ }^{1}$.

O traumatismo facial é considerado um problema de saúde pública relacionado a morbidades, perdas de função $0^{2}$ desfigurações ${ }^{2,3}$. A etiologia e incidência do trauma facial variam de um país para outro devido à existência de diferentes fatores locais, culturais e sociais ${ }^{2}$. Intimamente ligado ao trauma facial está o trauma dental, com alta prevalência, e por isso tem sido considerado um problema mundial $^{4}$.

Os casos de avulsão dentária geram a perda do órgão dentário, uma mutilação muitas vezes irreparável, pois causam o deslocamento por completo para fora de seu alvéolo, ocorrendo rompimento total do ligamento periodontal e do feixe vásculo-nervoso, onde a rapidez no tratamento favorece $o$ prognóstico $^{4,5}$.

Graduado em Odontologia, Unisul, Tubarão, SC, Brasil.

Graduanda em Odontologia, Unisul, Tubarão, SC, Brasil.

** Graduada em Farmácia, Docente da Universidade de Brasília.

*** Graduado em Odontologia, Docente da Universidade do Sul de Santa Catarina. 
Tendo em vista que o sucesso do reimplante depende do tempo de armazenamento do dente, do meio em que foi armazenamento e de sua correta manipulação, a negligência ou a demora no tratamento do traumatismo dental afeta a qualidade de vida dos pacientes sobremaneira quanto atinge os dentes anteriores, pois podem afetar a função, a atratividade física e a auto-estima do paciente, interferindo no comportamento e sucesso pessoal ${ }^{1,4,6}$.

A redução da morbidade e sequelas de lesões produzidas por acidentes violentos é proporcional ao tempo decorrido entre o acidente e admissão hospitalar ${ }^{4-6}$. Isto posto é de suma importância que o profissional que atua na linha de frente nestas situações esteja capacitado para o atendimento. Diante disto, o objetivo desta pesquisa foi avaliar profissionais que trabalham em serviço de atendimento de urgência de uma região de Santa Catarina, a respeito de avulsão e reimplante dental em pacientes com trauma facial.

\section{Sujeitos e método}

Este estudo caracterizou-se por um estudo transversal aprovado no CEP com o número 604.855-0/2014 com um total de 101 socorristas que atuam em serviço de urgência de uma região de Santa Catarina. Como critério de inclusão foram abrangidos todos os profissionais registrados como socorristas no serviço de atendimento de urgência de uma região de Santa Catarina, exercendo a função e que assinaram o termo de consentimento livre e esclarecido. Um questionário adaptado de Santos et $\mathrm{al.}^{7}$ (2006) com questões sócio-demográficas e de conhecimento sobre trauma facial, avulsão e reimplante dental foram aplicados aos participantes da pesquisa. As variáveis aplicadas a partir do questionário de $\operatorname{Santos}^{8}$ (2008) exploraram dados como: 1) qual o objetivo do atendimento inicial do socorrista à vitima; 2) se no atendimento ao paciente com trauma de face é investigado a existência de prótese dental ou fratura de dente natural; 3) se durante a formação ou treinamento os socorristas receberam informações sobre trauma dental, avulsão e/ou reimplante dental; 4) se o socorrista já teria atendido algum caso de trauma dental e/ou avulsão dental; 5) se o dente avulsionado poderia ser reimplantado e caso a resposta for sim, quem poderia realizar este procedimento; 6) qual o tempo máximo ideal que o dente pode ficar fora da boca antes do seu reimplante; 7) como proceder em uma situação de trauma facial com avulsão dental e presença do dente avulsionado. Os dados coletados foram inseridos numa planilha do Excel e exportados para o Software SPSS $20.0^{\circledR}$ para análise. Foi realizada a análise descritiva dos resultados, sendo adotadas as medidas de tendência central e dispersão para as variáveis numéricas e números absolutos e proporções para as variáveis categóricas.

\section{Resulltados}

Um total de 101 indivíduos participaram da pesquisa; a média de idade dos pesquisados foi de 32 anos e a média de tempo de serviço nas instituições é de nove anos e sete meses. A maioria dos entrevistados $(89,1 \%)$ era do sexo masculino.

Quando questionados sobre o atendimento inicial a uma vítima de trauma, 97\% ( $\mathrm{n}=98)$ dos entrevistados responderam que ao atender um acidente auxilia a vítima e tenta evitar consequências danosas no atendimento e no transporte, e quando há trauma de face, $71,3 \%(\mathrm{n}=72)$ dos participantes afirmaram buscar informação a respeito do uso de prótese dental e fratura dos dentes naturais.

A pesquisa ainda revelou que $74,3 \%(n=75)$ dos socorristas já atenderam casos de trauma dental. Entretanto apenas 38,6\% ( $n=39)$ dos entrevistados afirmaram já ter recebido alguma orientação acerca deste assunto durante sua formação.

Também com relação ao conhecimento sobre avulsão dental durante sua formação, 79,2\% (n=80) dos entrevistados responderam não ter recebido. Mesmo assim, 52,5\% (n=53) afirmam já ter atendido no mínimo um caso de avulsão dental.

A maioria dos entrevistados, $73,3 \%(\mathrm{n}=74)$, possui o conhecimento de que um dente avulsionado pode ser reimplantado, embora mais da metade, $56,4 \%(\mathrm{n}=56)$, indique que somente o dentista possa realizar este procedimento. Os entrevistados não formaram um consenso quanto ao tempo ideal do dente fora do alvéolo. A Tabela 1 evidencia os resultados.

Tabela 1 - Resultado do questionamento sobre o tempo de permanência de um dente fora do alvéolo referente a pesquisa realizada numa região de Santa Catarina, 2014

\begin{tabular}{l|c|c}
\hline $\begin{array}{c}\text { Tempo do Dente } \\
\text { Fora do Alvéolo }\end{array}$ & $\begin{array}{c}\text { Número de } \\
\text { Socorristas (n) }\end{array}$ & Percentual (\%) \\
\hline Imediato & 25 & 25,0 \\
1 \% Hora & 12 & 12,0 \\
1 Hora & 21 & 21,0 \\
6 Horas & 13 & 13,0 \\
Não Sabe & 29 & 29,0 \\
\hline
\end{tabular}

Apenas 19 (18,8\%) entrevistados afirmam que um dente avulsionado deve ser irrigado e colocado em solução salina ou na boca do paciente. A maioria dos entrevistados, $68,3 \%(\mathrm{n}=68)$, indica alguma forma de armazenar o dente avulsionado, outros $31 \%$ ( $\mathrm{n}=32$ ) não sabem ou não indicaram nenhuma forma de armazenamento. A Tabela 2 mostra os diferentes locais de armazenamento citados pelos entrevistados. 
Tabela 2 - Locais de armazenamento do dente avulsionado para posterior reimplante citados pelos entrevistados do serviço de atendimento de urgência de uma região de Santa Catarina, 2014

\begin{tabular}{l|c|c}
\hline $\begin{array}{c}\text { Local de Armazenamento do } \\
\text { Dente }\end{array}$ & $\begin{array}{c}\text { Número de } \\
\text { Socorristas (n) }\end{array}$ & $\begin{array}{c}\text { Percentual } \\
(\%)\end{array}$ \\
\hline $\begin{array}{l}\text { Recipiente com Água } \\
\text { Recipiente Vazio ou Enrolado } \\
\text { em Guardanapo }\end{array}$ & 22 & 22,0 \\
$\begin{array}{l}\text { Recipiente com Leite } \\
\text { Recipiente com Soro ou Em } \\
\text { Boca com Saliva }\end{array}$ & 10 & 20,0 \\
Não Sabe & 17 & 10,0 \\
\hline
\end{tabular}

Ao tentar reimplantar o dente, apenas 25,7 $\%(\mathrm{n}=26)$ dos entrevistados busca alinhá-lo junto aos dentes vizinhos, manipulando cuidadosamente, não tocando na raiz e procura serviço profissional.

\section{Discussão}

Os estudos apontam que os índices de trauma dental decorrentes de acidentes têm crescido nos últimos anos, principalmente acidentes de transito ${ }^{8,9}$. $\mathrm{Na}$ maioria destes casos o socorrista é o primeiro profissional a atender estas ocorrências. Diante dessa informação é essencial, ainda que não seja sua principal área de atuação, que estes detenham o conhecimento, mesmo que generalista, sobre o tema para impedir ou diminuir ações errôneas. Os dados obtidos evidenciam que os socorristas de uma região de Santa Catarina tiveram informação insuficiente durante sua formação, embora grande parte já tenha prestado atendimento em alguma situação envolvendo este tipo de trauma.

Embora pesquisas apontem as quedas, os acidentes automobilísticos e a violência como fatores etiológicos das avulsões dentais, não identificam o responsável pelo primeiro atendimento à vítima ${ }^{8-10}$. Mas como é notória a relação entre trauma facial, trauma dental e, por consequência, avulsão, fica subentendido que este atendimento de emergência é realizado na maioria das vezes pelo socorrista. Desta forma, reforça-se a necessidade deste profissional estar capacitado acerca dos procedimentos para um melhor prognóstico. O grau da lesão e da contaminação do ligamento periodontal, canal radicular e alvéolo dentário determinarão a forma de reparo periodontal após o reimplante, que vem a se relacionar, em maior ou menor intensidade às reabsorções radiculares, tendo em vista que quanto mais rápido for o reimplante, maior a probabilidade de se manter vitais as células do ligamento periodontal ${ }^{11-13}$.

Os resultados obtidos neste trabalho demonstram que, de forma empírica, 34 (33,66\%) dos socorristas de uma região de Santa Catarina buscaram algum tipo de informação referente à presença de trauma dental frente a um atendimento de vítima com trauma de face.
Não obstante a viabilidade de um reimplante dental seja discutida na literatura, tendo em vista o avanço da odontologia nas áreas de próteses e dos implantes, o reimplante nos casos de avulsão dental deve ser a opção primária de restauração da estética e função, desde que respeitados os princípios biológicos para o sucesso, mesmo que a longo prazo possa ocorrer a perda do elemento dental. $\mathrm{O}$ atendimento emergencial para dentes traumatizados é fundamental para o sucesso do tratamento. Perder um dente resulta em uma situação de difícil tratamento, causando impactos psicológicos e financeiros na vida do indivíduo, por isso o atendimento imediato é tão importante para reduzir tais consequências. A literatura reforça a importância dos cuidados com o dente avulsionado e o percentual de insucesso quando os protocolos de reimplante não são seguidos ${ }^{5,10-13}$.

Não houve consenso entre as respostas com relação ao tempo de permanência do dente fora do alvéolo. Cinquenta e oito $(57,4 \%)$ socorristas indicaram que o reimplante dentário deve ocorrer até 1 hora após o trauma, resultados que corroboram com a literatura para o sucesso do procedimento $0^{5,11,14}$.

Apesar de 69 (68, 31\%) dos socorristas terem indicado alguma forma de armazenamento, resultados que se equiparam ao encontrado na literatura com outros grupos pesquisados ${ }^{4-7,11-13,15,16}$, a maioria indicou um recipiente com água, local contraindicado pela Associação Internacional de Trauma Dental $(\mathrm{IADT})^{5}$, pois leva à desidaratação do tecido dentário e morte das células do ligamento periodontal.

Os dados desta pesquisa demostram que os socorristas sabem que um dente pode ser reimplantado. O principal desconhecimento é sobre quem pode realizar tal procedimento, porém salientam que diante de um dente avulsionado, ao reimplantá-lo, deve-se buscar o seu alinhamento junto ao dente vizinho, manipulando-o cuidadosamente e sem contato com a raiz sendo encaminhando o mais breve possível ao serviço profissional, concordando com protocolo preconizado pela IADT ${ }^{5}$.

A literatura evidencia que há um protocolo estabelecido e reconhecido por profissionais da área de odontologia como o ideal nos casos de avulsão dental $^{4,11,16,17}$, porém é pouco difundido a outros grupos profissionais, em especial aos que primeiro atendem estes casos, como demonstrado neste trabalho. Talvez tal fato seja um dos responsáveis pelo insucesso de alguns reimplantes dentais, como evidenciado pela literatura ${ }^{11,17,18 .}$

Estudos $^{14,16}$ demonstram que grupos profissionais, após receberem informação a respeito de trauma, aumentaram suas médias de acerto nas respostas significativamente. Isto demonstra que investir na transmissão de conhecimento a respeito do protocolo correto aumenta as chances de sucesso no tratamento. $\mathrm{O}$ socorrista não pode ser o elo fraco neste ciclo, devendo adquirir conhecimento quanto 
à avulsão dental e suas consequências, para que de forma adequada possa realizar o atendimento e aumentar as chances de sucesso no futuro tratamento.

Sugere-se que novos trabalhos, com uma amostra que expresse a totalidade dos socorristas do estado, sejam realizados, para que desta forma pontue-se com significado estatístico o grau de conhecimento. Mesmo que ainda não seja possível incluir na formação destes profissionais, o conteúdo específico sobre o tema, o conhecimento pode ser repassado através de palestras, reciclagem e grupos de discussão para que assim estes possam ter subsídio para o atendimento correto, tendo em vista que os socorristas de modo geral são os primeiros a prestar 0 atendimento nos casos de trauma facial ${ }^{17,18}$.

Os dados obtidos com este trabalho, ainda que demonstrem o insuficiente conhecimento frente a casos de avulsão dental, permite destacar que o contato com a informação ocorreu em alguns casos. Pode-se observar também que os pontos onde há maior dúvida sobre a conduta é frente ao armazenamento, tempo fora do alvéolo e reimplante, pontos também conflitantes em outros grupos pesquisados ${ }^{4,7,8,13,15}$. Reforça-se assim que cursos de capacitação e ou reciclagem sejam realizados com estes profissionais com vistas à padronização da conduta/protocolo ${ }^{19,20}$.

\section{Conclusão}

Após a realização deste estudo foi possível observar que o conhecimento dos profissionais que trabalham em serviço de atendimento de urgência de uma região de Santa Catarina sobre avulsão e reimplante dental em vítimas de trauma facial é insuficiente. Também foi verificado que os profissionais pesquisados não têm capacitação adequada ao atendimento de casos de avulsão. Entretanto, como os protocolos de atendimento a estas situações já são reconhecidos na literatura, sugere-se a reciclagem ou a capacitação destes profissionais com a inserção deste conteúdo para uma padronização do atendimento.

\section{Abstract}

Objective: to evaluate the level of knowledge of emergency physicians regarding the care on tooth avulsion and replantation cases. Subjects and method: cross-sectional study carried out through interviews with emergency physicians working in an emergency care service in a region of the state of Santa Catarina, Brazil. Results: 101 collaborators were interviewed, of which $74.3 \%$ (75) affirmed having treated cases of dental trauma and $52.5 \%$ (53) of tooth avulsion, while only $38.6 \%$ (39) of respondents referred having received some instruction on dental trauma and $20.8 \%$ (20) on avulsion during their training. From the respondents, 74 (73.3\%) know that an avulsed tooth may be replanted, only 19 (18.8\%) affirmed that the element should be irrigated and placed in saline solution or in the patient's mouth, and $69(68.3 \%)$ reported some form of storage for the avulsed tooth. Conclusion: the knowledge of professionals working in the emergency care service studied regarding the care for patients who have suffered dental trauma is insufficient for proper care.

Keywords: Facial traumas. Dental traumas. Tooth Avulsion. Dental replantation.

\section{Referências}

1. Piva F, Potter IG, Sari GT, Klein-Júnior CA, Coelho-De Souza FH. Atendimento de Urgência Frente ao Traumatismo Alvéolo Dentário - Relato de Caso Clínico. Rev Assoc Paul Cir Dent 2013: 67(3):224-8.

2. Wulkan M, Parreira JG, Botter DA. Epidemiologia Do Trauma Facial. Rev Assoc Med Bras 2005; 51(5):290-5.

3. Brasileiro BF, Passeri LA. Epidemiological Analysis Of Maxillofacial Fractures In Brasil: A 5-Year Prospective Study. Oral Surg Oral Med Oral Pathol. Oral Radiol Endod 2006; 102(1):28-34.

4. Jetro V, Morais HHA, Dias TGS, Barbalho JCM, Luceno ES. Traumatismo Dentoalveolar: Nível de Conhecimento e Conduta de Urgência dos Bombeiros do Município de Caicó-RN. Rev Cir Traumatol Buco-Maxilo-Fac Camaragibe 2013; 13 (2):101-8

5. Andersson L, Andreasen JO, Day P, Heithersay G, Trope M, Diangelis AJ et al. International Association of Dental Traumatology guidelines for the management of traumatic dental injuries: 2. Avulsion of permanent teeth. Dent Traumatol 2012; 28 (2):88-96

6. Schatz JP, Hakeberg M, Ostini E, Kiliaridis S. Prevalence Of Traumatic Injuries To Permanent Dentition And Its Association With Overjet In A Swiss Child Population. Dent Traumatol 2012; 29(2):110-14.

7. Santos MESM. Conhecimento De Pais Ou Responsáveis Sobre Avulsões De Dentes Permanentes. [Dissertação de Mestrado]. Porto Alegre: Pontifícia Universidade Católica; 2006. Disponível em http://hdl.handle.net/10923/475

8. Santos CML, Musseb JO, Cordeiro IS, Martins TMN. Estudo Epidemiológico Dos Traumas Bucomaxilofaciais Em Um Hospital Público De Feira De Santana, Bahia De 2008 A 2009. Rev Baiana de Saúde Pública 2012; 36(2):502-13.

9. Zhou HH, Ongodia D, Liu Q, Yang RT, Li ZB. Dental Trauma In Patients With Maxillofacial Fractures. Dental Traumatology 2013; 29(4):285-90.

10. Zhang X, Gong Y. Characteristics Of Avulsed Permanent Teeth Treated At Beijing Stomatological Hospital. Dent Traumatol 2011; 27(5):379-84.

11. Werder P, Arx TV, Chappuis V. Treatment Outcome Of 42 Replanted Permanent Incisors With A Median Follow-Up Of 2.8 Years. Schweiz Monatsschr Zahnmed 2011; 121(4):31220.

12. Costa ED, Queiroz FS, Nóbrega CBC, Leite MS, Nóbrega WFS, Almeida ER. Trauma Dentário na Infância: Avaliação da Conduta dos Educadores de Creches Públicas de Patos-PB. Rev Odontol UNESP 2014; 43(6):402-8.

13. Santana NM. Avaliação Do Conhecimento Sobre A Conduta Emergencial Do Trauma Dental De Profissionais Do Futebol Em Curitiba, Brasil. [Dissertação de Mestrado]. Curitiba: Universidade Federal do Paraná; 2012. 
14. Azami-Aghdash S, Azar FE, Azar FP, Rezapour A, Moradi-Joo M, Moosavi A et al. Prevalence, Etiology and Types of Dental Trauma in Children and Adolescents: Systematic Review and Meta-Analysis. Med J Islam Repub Iran 2015; 29(4):234.

15. Alves LSB, Freitas VJG, Rosendo RA, Gominho LF, Sarmento TCAP. Avaliação do Conhecimento de Professores do Ensino Fundamental da Rede Particular Sobre Atendimento Imediato de Vítima de Traumatismo Dental. Rev da Fac Odontol Univ Passo Fundo 2015; 20(3):302-7.

16. Al-Shamiri HM, Alaizari NA, Al-Maweri S, Tarakji B. Knowledge and Attitude of Dental Trauma Among Dental Student in Saudi Arabia. Eur of Dentistry 2015; 9 (4):518-22.

17. Raoof M, Shokouhinejad N, Izadi A, Nourzadeh M, Afkham A, Forghani FR et al. Long-Term Effect Of An Educational Intervention Regarding Dental Trauma First Aid: A Phase II Study. Dental Traumatology 2013; 124(32):36-9.

18. Mahmoodi B, Rahmi-Nedjat R, Weusmann J, Azaripour A, Walter C, Willeshausen B. Traumatic Dental Injuries In a University Hospital: A Four-Year Retrospective Study. BMC Oral Hearth 2015; 15:139.

19. Grewal N, Shangdlar GD, Samita G. Efficacy of a Comprehensive Dental Education Program Regarding Management of Avulsed Permanent Teeth as a Valid of Increased Success Rate of Treatment of Avulsion in a North Indian Population. Contemp Clin Dent 2015; 6(4):447-82.

20. Baginska J, Rodakowska E, Milewski R, Borawska-Wikzynska M, Kierklo A. Polish School Nurses's Knowledge of The First-Aid in Tooth Avulsion of Permament Teeth. BMC Oral Hearth 2016; 16:30.

\section{Endereço para correspondência:}

Glaucia Helena Faraco de Medeiros

Av. José Acácio Moreira, 787

88704-900 Tubarão/SC Brasil

E-mail: glaucia.medeiros@unisul.br

Recebido: 04/01/2017. Aceito: 02/04/2017. 